\title{
Influence of the Anode Material and the Flushing Gas on the Dry Electrical Discharge Machining
}

\section{Process}

\section{Journal Article}

Author(s):

Roth, Raoul; Beck, Lukas; Balzer, Hartmi; Kuster, Friedrich; Weingärtner, Eduardo; Wegener, Konrad

Publication date:

2013-09

Permanent link:

https://doi.org/10.3929/ethz-a-009952539

Rights / license:

In Copyright - Non-Commercial Use Permitted

Originally published in:

International Journal of Automation Technology 7(5), https://doi.org/10.20965/ijat.2013.p0581 
Paper:

\title{
Influence of the Anode Material and the Flushing Gas on the Dry Electrical Discharge Machining Process
}

\author{
Raoul Roth*, Beck Lukas*, Hartmi Balzer**, \\ Friedrich Kuster*, Eduardo Weingärtner ${ }^{* * *}$, Konrad Wegener ${ }^{*, * * *}$ \\ * Intitute of Machine Tools and Manufacturing, ETH Zürich \\ CLA G4, Tannenstrasse 3, 8092 Zürich, Switzerland \\ E-mail: raoul.roth@iwf.mavt.ethz.ch \\ **Balzer Technik AG \\ Route de l'industrie 65, 1564 Domdidier, Switzerland \\ *** Inspire, Transfer Institute for Mechatronic Systems and Manufacturing Technology, Zürich, Switzerland \\ [Received April 22, 2013; accepted August 9, 2013]
}

In the last years dry electrical discharge machining (DEDM) has been proposed as an alternative to the traditional EDM. The main reason for these efforts is the absence of a liquid dielectric which results in a simpler and environmentally friendly process. This paper presents measurements of the material removal rate in function of different tool electrodes, work piece materials and flushing gases put in relation with the breakdown behavior of the process. Evaluation of absolute and current specific material removal rate are presented. The data show a big influence on the material removal rate depending on the combination of work piece material and flushing gas. Two different effects are observed, the first enhancing the removal per spark and the second one reducing the short circuiting occurrence. The share of these two effects on the enhancing of the absolute material removal rate also differs in function of the work piece material. It is suggested that the chemical reaction strongly influences the process in two different ways, on one hand releasing a surplus of energy and on the other hand changing the debris particles' properties.

Keywords: Dry Electrical Discharge Machining (DEDM), material removal rate (MRR), removal mechanism, electrical breakdown

\section{Introduction}

Electrical Discharge Machining (EDM) is a well known and established machining process characterized by its high precision and ability to machine materials considered hard to machine. Nevertheless the removal mechanism of the process is still disputed and beside a good empirical knowledge there is still poor insight of the physical phenomena according to [1]. Dry Electrical Discharge Machining (DEDM) is a new process based on the traditional EDM with the particularity that gaseous dielectric is utilized instead of liquid dielectric. The advantage in respect to traditional EDM is first of all that no liquid is needed for the process. This allows a simpler EDM machine which does not need to take care of keeping the liquid inside. Another advantage is, that in comparison to die sinking EDM usually performed in mineral oil, the process is environmental friendlier. Other advantages as higher removal rate and very little tool wear ratio have been attributed to DEDM in [2] and [3]. Furthermore the capability to combine EDM with other processes, to design process chains with EDM can be enhanced.

In the traditional process the main function of the liquid dielectric is to isolate electrode and work piece, to cool them and to flush the working gap ensuring a stable process. To ensure these functions without liquid dielectric some measures need to be taken in the dry version of the process. Kunieda and Yoshido [4] proposed to use a rotating tube electrode flushed from the inside, which became a standard for DEDM. The pressurized gas coming from the inside of the electrode carries away the eroded material particles from the working gap. The rotation of the electrode helps flushing creating a vorticity and leads to a more stable process. An enhancement of the material removal rate adding oxygen in a liquid dielectric has been shown by Kunieda and Furuoya already in [5]. In DEDM usually pure molecular oxygen is used as working gas because of its supportive effect on material removal rate. Further improvement of the process has been presented by Kunieda et al. in [6] using a piezoelectric actuator with the aim to avoid the high short circuiting probability due to the very narrow gap we have in DEDM. Zhang et al showed in [7] that assisting DEDM with ultrasonic vibration also improves the material removal rate. Efforts have been made as reported by [8] to characterize this new process and optimize it, trying to reach higher removal rates, lower tool electrode wear and better surface characteristics. A complete review of the research works in the field of DEDM till 2006 can be found in the work of Abbas et al [9]. The removal mechanism of EDM is a stochastic microscopic elementary phenomenon which is repeated at high frequencies and thus leads to a macroscopic predicable process. The models of EDM removal mechanism usually base on the thermal energy introduced into the work piece through the point where the plasma 
channel is in contact with the metal. Due to the little time and space scale as well as the stochastic nature of the process a physical understanding of the microscopic removal mechanism relied on experimental proof is very hard to achieve. The knowledge gained in the long research on EDM in liquids has been often transferred to the younger research on DEDM and used to explain the experimentally observed phenomena. But the analogy goes astray as the physical mechanisms behind the process are evidently not all the same as in wet EDM. For both EDM processes, dry or wet, it is important to have a stable gap control able to maintain the ideal distance from tool electrode to the work piece.

Parameters that influence the breakdown distance of the gap are mainly the applied voltage, the breakdown strength of the dielectric fluid, the pollution of the gap, the electrode surface, the material and the geometrical form of the tool electrode. In traditional EDM the breakdown voltage of the dielectric fluid is small enough that gaps from few micrometers till hundreds of micrometers are possible. On the contrary in DEDM only very narrow gaps are possible because of the high breakdown voltage of the gases applied. This is a big challenge for the technology because the small gap is harder to control. The servo control is not able to avoid short circuit on one side and longer ignition delay times on the other side. This paper shows how the work piece material and the flushing gas are crucial for the process stability and how they affect the removal rate not only due to the different physical properties leading to different removals per spark, but also due to the properties that affect the discharge behavior of the process. The different results presented here depend not only on these two individual parameters but show also a strong interdependency of the two. The aim of this work is to gain a better insight of the controlling factors of the process regarding the MRR and the stability of the DEDM process. For this reason not many results regarding surface integrity and tool wear are presented maintaining the focus on the removal mechanism.

\section{Materials and Methods}

\subsection{Experimental Set Up}

The experimental set up consists in a Spirit II EDM machine from AGIE. An additional spindle from 3R (3R6.300-EHS16) provides a high speed rotation of the tool electrode. High pressure gas is provided from gas bottles through a pressure regulator and a mass flow measurement system into the spindle and then from the inside of the tool electrode to the work gap. The setup is designed for tool electrode rotational speed up to 2000rpm and gas pressures up to 35 bars. The working gas can be easily changed by changing the gas bottle. In the DEDM experiments, that are presented here, only oxygen and air have been used as working medium. A LeCroy Wave Runner 44MXi-A digital oscilloscope is used to monitor and evaluate the voltage and the current signals over the time.

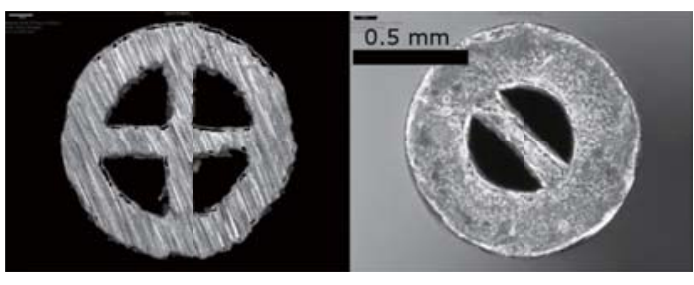

Fig. 1. Cross section of cemented carbide electrode (left) and copper electrode (right).

\subsection{Electrodes and Work Pieces}

The used electrode materials are pure copper and cemented carbide multichannel electrodes from Balzer Technik. The copper electrode is a two channel electrode and the cemented carbide is a four channel electrode. The cross sections are shown in Fig. 1. The outer diameter of both electrodes is 1 millimeter. The inner diameter of the copper electrode is 0.44 millimeters and the width of the division bar is 0.13 millimeters which results in a cross section of the copper electrode of 0.69 square millimeters. The cemented carbide electrode has an inner diameter of 0.6 millimeters and division bars with a width of 0.135 millimeters. This corresponds to a cross section of the cemented carbide electrode of 0.64 square millimeters. The multichannel electrode with two or four channels has mainly two advantages. On the one hand during rotation it curls the gas flow more than a single channel electrode leading to a better flushing of the working gap. On the other hand, if a pure drilling operation is executed it avoids the formation of a gudgeon in the middle of the hole. The utilized work piece materials are the stainless steel SS304, cemented carbide, aluminum, copper and gold.

\subsection{Measurements}

During the erosions the machining time is measured and documented. The eroded volume is evaluated after the erosion with a 3 dimensional data set of the work piece, generated with an Alicona Infinite Focus microscope. Dividing the eroded volume by the erosion time the Material Removal Rate $(M R R)$ in $\mathrm{mm}^{3} / \mathrm{min}$ can be calculated.

The tool wear is measured directly after the erosion, measuring the difference of the electrode length before and after the erosion process $\Delta l$. Multiplying the worn length $\Delta l$ with the cross section area $A_{T}$ of the tool electrode the worn volume is obtained. The Tool Wear Ratio $(T W R)$ is calculated by dividing the worn volume of the electrode by the eroded volume of the work piece:

$$
T W R=\frac{A_{T} \cdot \Delta l}{V_{E}}
$$

During the erosion also the electrical behavior of the process is monitored and measured on the oscilloscope. A minimum of two seconds with a resolution of $20 \mathrm{MHz}$ of voltage and current signal over the working gap are stored 
for every erosion test. The stored data is analyzed evaluating the duration of the time in which normal discharges, arcing discharges, short circuits or ignition delays have occurred. In order to evaluate this data the voltage signal is scanned for negative pulses, which means signals that go below a defined value for a given time and then rise again. The duration of the scanned pulses is noted. In case of normal sparks this length of time corresponds to the sum of spark time $T_{S}$ and pause time $T_{P}$. Filtering several voltage levels and pulse lengths, a list of all the sparks, arcings and shorts with their respective length is extracted. The signal is considered to be a short when the voltage signal goes below $8 \mathrm{~V}$ and the measured pulse duration is at least $T_{1}=1.5\left(T_{S}+T_{P}\right)$. Arcing together with short times are listed when the voltage signal goes below $2 / 3$ of the open voltage $U$ and the pulse duration is at least also $T_{1}$. The spark time, arcing time and short times are listed together when the voltage goes below $2 / 3$ of the open voltage $U$ and the pulse duration is at least $T_{2}=2 / 3\left(T_{S}+T_{P}\right)$. The right combination of the measured times finally permits to educe the individual sparking, arcing and short circuit times. Putting the results in relation with the total measurement time of two seconds, the partitions of the occurred spark, arcs, shorts and ignition delay times can be calculated. Two seconds may look not representative for an erosion that can take several minutes, but in comparison to the regulation times of the working axis, which happens in the $\mathrm{kHz}$ range, it becomes clear that in a couple of seconds enough cycles have occurred.

The surface roughness $R_{a}$ has been measured picturing the bottom of the drilled holes with Alicona Infinite Focus microscope. From the optically generated $3 \mathrm{D}$ data set a value for $R_{a}$ is evaluated conform to ISO 4287 .

\section{Experiments}

Two sets of experiments are presented here. The first one aims to show the difference between cathode and anode material regarding process stability and $M R R$ and is described in section 3.1 and section 4.1 respectively. The aim of the second one is to show the influence of the flushing gas in combination to the work piece material on the $M R R$ and it's described in section 3.2 and section 4.2 respectively. It is well known, but also been observed in this work, that in DEDM an important removal happens at the anode and only a poor one at the cathode independently from the chosen erosion parameters. Therefore in the work presented here all experiments have been conducted with negative tool polarity. The rotational speed of the electrode has been set at the maximum possible value of the spindle, since the maximum $M R R$ in function of the rotational speed is observed at this value.

\subsection{Comparison of Different Anode and Cathode Materials in DEDM with Oxygen}

As experiments vertical drilling of holes of at least 1 millimeter depth are performed. To see how the tool
Table 1. Fixed DEDM parameters of the experiments in series 1 .

\begin{tabular}{|l|c|c|}
\hline Parameter & Symbol & Value \\
\hline Tool electrode polarization & & negative \\
\hline Open voltage $[\mathrm{V}]$ & $U$ & 250 \\
\hline Current $[\mathrm{A}]$ & $I$ & 6 \\
\hline Spark time $[\mu \mathrm{s}]$ & $T_{S}$ & 15.4 \\
\hline Pause time $[\mu \mathrm{s}]$ & $T_{P}$ & 15.4 \\
\hline Flushing pressure $[\mathrm{bar}]$ & $p$ & 20 \\
\hline Electrode rotation speed $[\mathrm{rpm}]$ & $\Omega$ & 2000 \\
\hline Flushing gas & & Oxygen \\
\hline
\end{tabular}

Table 2. Material combination of the experiments in series 1 .

\begin{tabular}{|l|c|c|}
\hline Experiment & Tool electrode & Work piece \\
\hline 1 & $\mathrm{Cu}$ & $\mathrm{SS} 304$ \\
\hline 2 & $\mathrm{CC}$ & $\mathrm{SS} 304$ \\
\hline 3 & $\mathrm{Cu}$ & $\mathrm{CC}$ \\
\hline 4 & $\mathrm{CC}$ & $\mathrm{CC}$ \\
\hline
\end{tabular}

Table 3. Changed parameters for experiments in oil in series 1.

\begin{tabular}{|l|c|c|}
\hline Parameter & Symbol & Value \\
\hline Flushing pressure [bar] & $p$ & 1.5 \\
\hline Electrode rotation speed [rpm] & $\Omega$ & 40 \\
\hline Flushing fluid & & Oil \\
\hline
\end{tabular}

electrode and work piece material influences the material removal rate a fixed set of dry electrical discharge machining parameters and a single gas has been chosen - only the materials are changed. The main parameters are listed in Table 1. The electrical parameters have been chosen to guarantee a stable and not too aggressive process for the combination of several electrode materials. For this reason parameters as for example pause time $T_{P}$ and current $I$ are not set to reach the maximum of the removal rate. The combinations of the tool electrode and work piece materials which have been tested are listed in Table 2. The $M R R$, the discharge behavior, the current specific $M R R$ and the $T W R$ have been evaluated for this erosions. Only one trial per material combination has been conducted. For this reason no standard deviation can be evaluated.

To have a comparison of the discharge behavior in a liquid dielectric the same experiment has been conducted in oil. The aim was not to compare the material removal rate which has not been optimized for none of the two processes but to have a comparison of the electrical behavior of the two processes. For this reason the same parameters which have been used in DEDM have been used for the oil erosion. Just the rotational speed and the flushing pressure of the working fluid have been changed because the experimental set up for erosion in oil was not capable to reach the same values. In Table 3 the values of the pa- 
Table 4. Material combination of the experiments in series 2.

\begin{tabular}{|l|c|c|c|}
\hline Experiment & Tool electrode & Work piece & Gas \\
\hline 1 & $\mathrm{Cu}$ & $\mathrm{SS} 304$ & Air \\
\hline 2 & $\mathrm{Cu}$ & $\mathrm{SS} 304$ & Oxygen \\
\hline 3 & $\mathrm{Cu}$ & $\mathrm{Cu}$ & Air \\
\hline 4 & $\mathrm{Cu}$ & $\mathrm{Cu}$ & Oxygen \\
\hline 5 & $\mathrm{Cu}$ & $\mathrm{Al}$ & Air \\
\hline 6 & $\mathrm{Cu}$ & $\mathrm{Al}$ & Oxygen \\
\hline 7 & $\mathrm{Cu}$ & $\mathrm{Au}$ & Air \\
\hline 8 & $\mathrm{Cu}$ & $\mathrm{Au}$ & Oxygen \\
\hline
\end{tabular}

Table 5. Fixed EDM parameters of the experiments in series 2.

\begin{tabular}{|l|c|c|}
\hline Parameter & Symbol & Value \\
\hline Tool electrode polarization & & negative \\
\hline Open voltage $[\mathrm{V}]$ & $U$ & 250 \\
\hline Current $[\mathrm{A}]$ & $I$ & 7 \\
\hline Spark time $[\mu \mathrm{s}]$ & $T_{S}$ & 15.4 \\
\hline Pause time $[\mu \mathrm{s}]$ & $T_{P}$ & 7.5 \\
\hline Flushing pressure $[\mathrm{bar}]$ & $p$ & 20 \\
\hline Electrode rotation speed $[\mathrm{rpm}]$ & $\Omega$ & 2000 \\
\hline
\end{tabular}

rameters which have been different are listed. For these erosions in oil three repetitions have been done for every material combination. The error bars visible in section 4 is the standard deviation of the three results.

\subsection{Comparison of Different Anode Material in Combination with Different Flushing Gases for DEDM}

To gain insight also into the influence of gases and especially the mutual influence of gas and work piece material on discharge phenomena and $M R R$ the same vertical drilling erosions are performed also with different anode materials in combination with different flushing gases.

Essentially four work piece materials (steel, copper, aluminum and gold) are eroded with a copper electrode and two different flushing gases (air and oxygen). All the combinations of the experiments are listed in Table 4. Erosion parameters with a little more energy are chosen for these experiments. The reason is to have not too small $M R R$ for the erosion with air so a larger current and a shorter pause time are chosen. The applied parameters are listed in Table 5. The $M R R$, the discharge behavior, the current specific $M R R$ and the resulting surface roughness have been evaluated for these erosions. For the experiments with copper, aluminum and gold as work piece three repeats have been conducted. The results presented in section 4.2 are the mean value and the error bar is the standard deviation of the repeats. The erosions in stainless steel have been made in combination with other experiments with no repeats of the single trials. For this reason no standard deviation can be calculated and no error bar

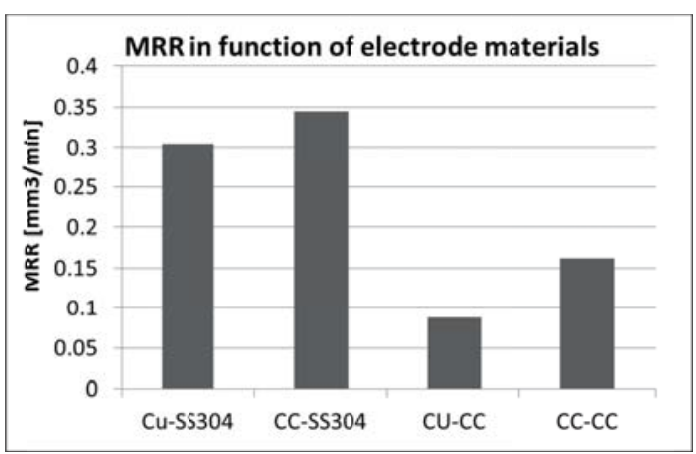

Fig. 2. Material removal rate in function of cathode and anode material combinations.

is shown in the graphs for the erosions in stainless steel. Also no surface roughness can be evaluated for stainless steel since the work pieces have been used for other destructive measurements. Nevertheless the comparison of the $M R R$ and the process stability is interesting and therefore presented in section 4.2 with the other materials.

\section{Results}

\subsection{Results with Different Anode and Cathode Material with Oxygen}

\subsubsection{Material Removal Rate}

The result of the experiment shows a little higher material removal rate when cemented carbide tool electrode is used instead of the copper electrode. In Fig. 2 the measured material removal rate is represented in dependence on the several material combinations. When cemented carbide is used as work piece the material removal rate is clearly less than the one with stainless steel. So it becomes obvious that the work piece material has a major influence on the material removal rate. On the other hand the influence of the tool electrode material on the material removal rate is very small in comparison to the influence of the work piece material. A strong dependency of the work piece material and a poor one of the tool electrode on the $M R R$ are expected, since the material properties such as melting temperature and heat conduction coefficient of the work piece and not the one of the tool electrode are decisive to the crater volume created by single sparks and consequently to the removal rate. This is reasonable as long as we can assume the same discharge frequency and input energy. In section 4.1.3 and section 4.1.4 the discharge behavior and the current specific material removal rate of the same erosions are presented. The results show big differences in the discharge behavior and therefore in the effective input energy. Considering this we have to be very careful deducing the reasons of the different measured MRR. An extensive discussion on this topic will follow in section 5.1 and 5.2. 


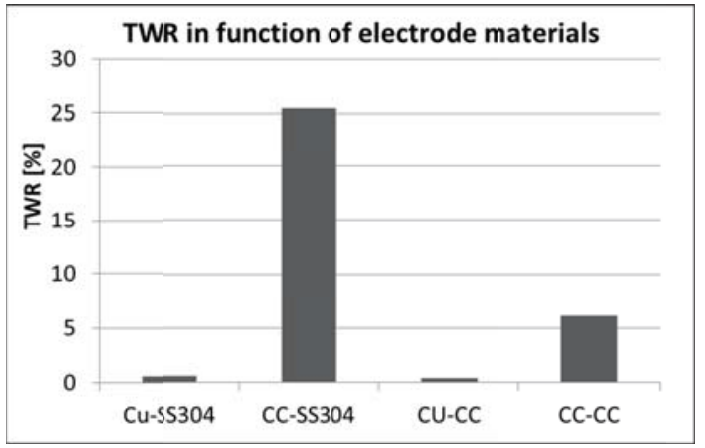

Fig. 3. Tool wear ratio in function of cathode and anode material combinations.

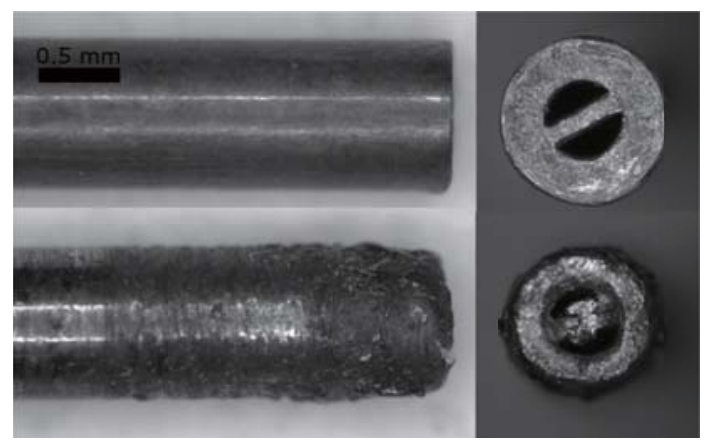

Fig. 4. Example of new and worn copper multichannel electrode.

\subsubsection{Tool Wear Ratio}

In Fig. 3 the tool wear ratio is represented in dependence on the several materials that have been combined. It is observed that $\mathrm{CC}$ has a bigger $T W R$ then $\mathrm{Cu}$. The reason for this behavior might be the different heat conduction coefficient of the two materials. The copper withdraws heat faster from the working area reducing the erosion of the tool electrode. The reason for the difference between the CC-SS304 and the CC-CC tool wear ratio can be found looking at the discharge behavior (see section 4.1.3).

With cemented carbide work piece the frequency of the shorts becomes larger, so the ratio of time where energy is converted in the gap drops. For this reason the cemented carbide tool electrode has more time to take away the heat and wears less than while eroding SS304.

In Fig. 4 an example of a dressed copper electrode and a worn one are shown. The length wear of the electrode, i.e. the frontal shortening which is happening during the erosion, is not visible in these pictures because we fitted the electrode in the image. The shortening is measured right after the erosion and used to calculate the $T W R$ how described in section 2.3 and showed in Fig. 3. In Fig. 4 we can observe a rounding of the corner in front of the electrode as well as some attachments of material on the frontal area and on the sidewalls. These images shall be

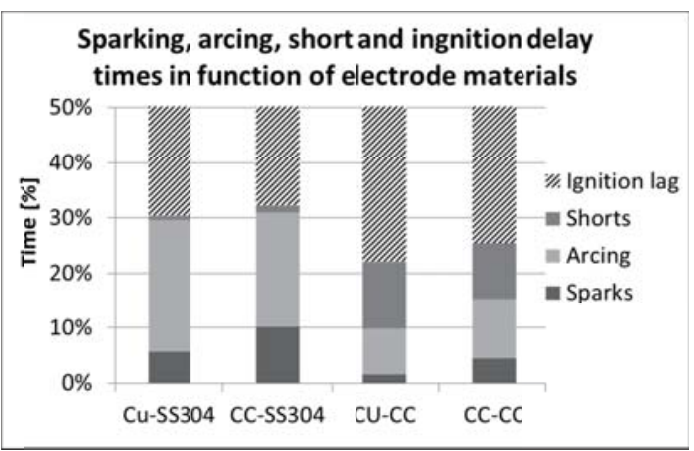

Fig. 5. Sparking, arcing, shorts and ignition delay times in function of anode and cathode material combinations for Dry Electrical Discharge Machining (DEDM).

an example of a worn electrode although the wear and the material attachment may look different in function of flushing gas or work piece material.

\subsubsection{Discharge Behavior}

In Fig. 5 the sparking, arcing, short circuit and ignition delay times are represented in function of the several material combinations. It has to be noted that that the $Y$ axis is displayed only till $50 \%$ hiding the $50 \%$ where only ignition lag occur. This is done to see better the significant part of the graph, but the total sum of the bars is always $100 \%$ and the not displayed part belongs to the ignition lag. The setting of the erosion parameters should lead to a theoretical relative ignition lag time of 70 (this value is valid for all the experiments presented here). When only few short circuits occur, the rest of the time will be divided between sparks and arcs with a total of about $30 \%$. But if many short circuits occur the machine retracts the electrode trying to avoid them. This reaction leads to a bigger ignition lag time and the summed time of sparks, arcs and short circuits drops to smaller values than the theoretical $30 \%$. Here it is notable - as has already been shown for the material removal rate - that there is a major influence from the work piece material and only a small influence from the tool electrode material. For erosion like the two in SS304 where few short circuits occur and a value near the theoretical ignition lag is reached we speak about a stable process. On the other side, as we can observe for the erosions in $\mathrm{CC}$ where many short circuits occur and the ignition lag time is rising we speak about a less stable process.

\subsubsection{Current Specific Material Removal Rate}

For manufacturing specific material removal rates are always defined by dividing the $M R R$ by some effort variable. A current Specific Material Removal Rate (SMRR) is defined by dividing the $M R R$ by the time averaged current value $I_{a}$ :

$$
I_{a}=I \frac{T_{S}}{T_{S}+T_{P}}\left(\tau_{A}+\tau_{S}\right) \quad \ldots . . . . .
$$




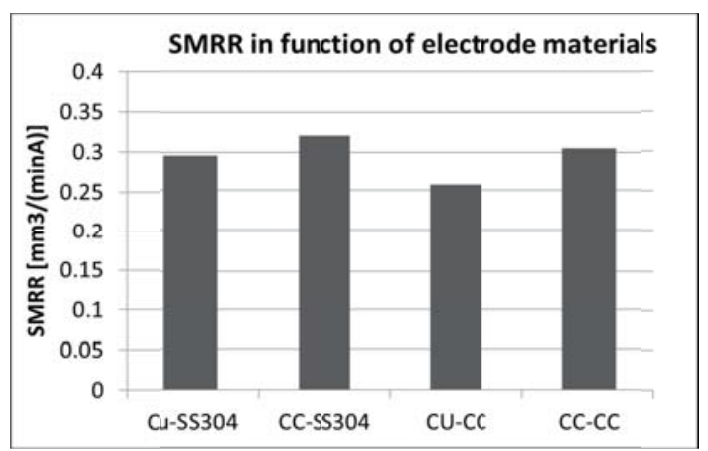

Fig. 6. Current specific material removal rate in function of cathode and anode materials.

The time averaged current is calculated with the relative sparking time $\tau_{S}$ and the relative arcing time $\tau_{A}$ because these are the only times, where the flowing current is supplying energy to the working gap. In case of short circuiting the gap voltage is vanishing, so no power is converted over the gap. In case of ignition delay obviously no power is converted too because the current is not flowing. The spark time $T_{S}$ divided by the sum of spark time $T_{S}$ and pause time $T_{P}$ is representing the duty cycle of the process. It should be noted that since the quantity of charges in one spark is given by the generator in function of the current $I$ and the discharge time $T_{S}$ and remains fixed during the process. The current specific material removal rate $S M R R$ is thus proportional to the removal per spark. In Fig. 6 the $S M R R$ is represented in function of several anode and cathode material combinations. It becomes clear that the current specific removal rate does not change significantly in function of the work piece material. In other words, also if the absolute $M R R$ shows clear differences in function of the anode material, there is quite no difference of removal per spark.

This confirms that the influence of the stability on the $M R R$ is much bigger than the influence due to the different physical properties of the work piece material, which should lead to different removals per spark. The influence of the anode material on stability is discussed further in section 5 .

\subsubsection{Discharge Behavior in Oil}

In Fig. 7 the discharge behavior in dielectric oil is presented. Also here a clear influence of the tool electrode and work piece material is noticeable. The negative tool electrode polarization which is not ideal for copper-steel erosion, the short pause time $T_{P}$ as well as the different flushing of the working gap lead to high short circuit rates. Because it is important to have the same polarization of the electrodes, the poor sparking and arcing times are taken into account by just look at the relative behavior in function of the materials. In contrast to the DEDM results the influence of work piece electrode material seems not to be so dominant.

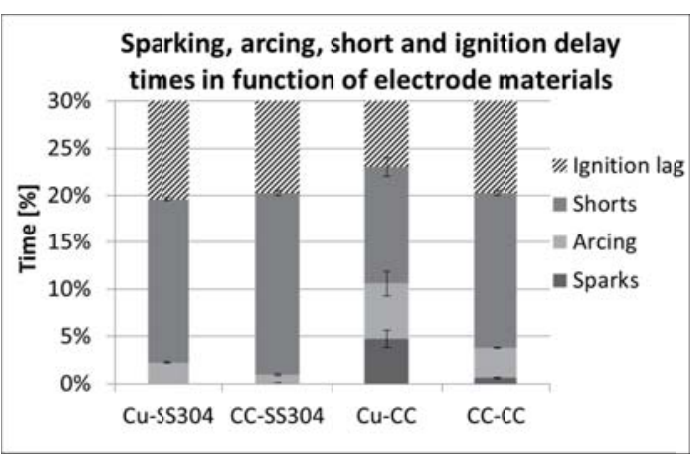

Fig. 7. Sparking, arcing, shorts and ignition delay times in function of cathode and anode material combinations for classical EDM in oil dielectric.

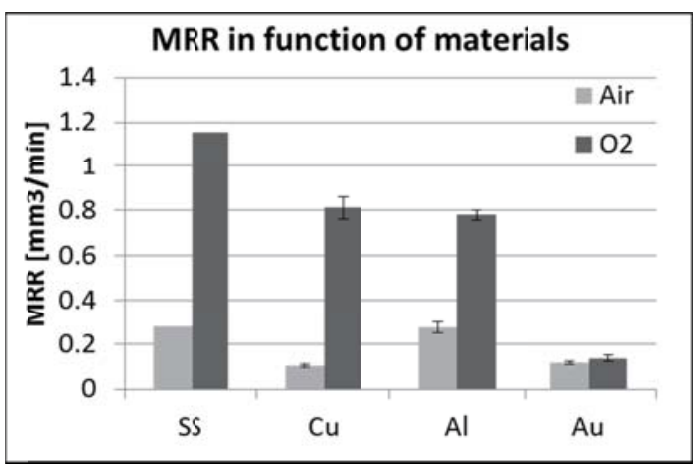

Fig. 8. Material removal rate in function of materials and different gases.

\subsection{Results for Different Anode Materials and Flushing Gases}

\subsubsection{Material Removal Rate}

In Fig. 8 the $M R R$ in function of different work piece materials and flushing gases can be found. The error bar of copper, aluminum and gold represents the standard deviation measured by repeating the experiments three times. We observe for all the measured materials but gold that the flushing gas has a strong influence on the MRR. This obviously correlates with the inert nature of gold. This evidence along with the further analysis of stability and current specific material removal rate allows interesting conclusions discussed in section 5 which go beyond the trivial differences between the materials and gases.

\subsubsection{Discharge Behavior}

The measurement of relative spark, arc short and ignition lag times also shows some clear differences in function of work piece and flushing gases. Surprisingly two different behaviors in function of the flushing gas can be distinguished. On one hand we have stainless steel and copper that strongly change discharge behavior when oxygen is applied as flushing gas instead of air. On the other hand we have aluminum and gold which stability 


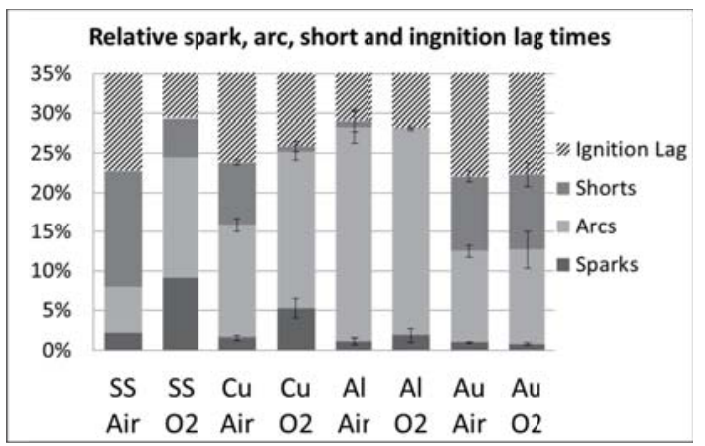

Fig. 9. Sparking, arcing, shorts and ignition delay times in function of different materials and gases.

seems not to be affected from the flushing gas. For SS304 and copper, there is a correlation between the material removal rate and the stability of the process but there is none for aluminum, where despite unaffected stability in function of the flushing gas, big differences in the $M R R$ are measured. The current specific material removal rate helps us interpreting the presented data.

\subsubsection{Current Specific Material Removal Rate}

Concerning the influence of flushing gas on SMRR work piece materials can be distinguished in three different classes of behavior.

1.) Gold shows no difference in the stability nor in the material removal rate, when changing the gas. No significant changes can be noted for the discharge frequency as well as the removal per spark.

2.) Aluminum shows no significant difference in function of the gas regarding the stability and the discharge frequency but a big difference for the absolute MRR and the SMRR.

This gives clear evidence, that oxygen as flushing gas enhances the removal per spark with respect to erosion with air, but doesn't change the discharge frequency.

3.) Steel and copper show a difference in the stability and also a difference in absolute and specific $M R R$. This means that the enhancement of the absolute $M R R$ of these two materials by larger oxygen content in the flushing gas is due to a larger removal per spark and a more stable process with a higher discharge frequency. The proportion of the influence of the two effects is different for the two materials. While for stainless steel the percentage of influence due to the stability or the removal per spark are roughly 70 and $30 \%$ respectively, for the copper they are 60 and $40 \%$. For aluminum 0 and $100 \%$ respectively are obtained, since only the removal per spark is influenced by the oxygen content.

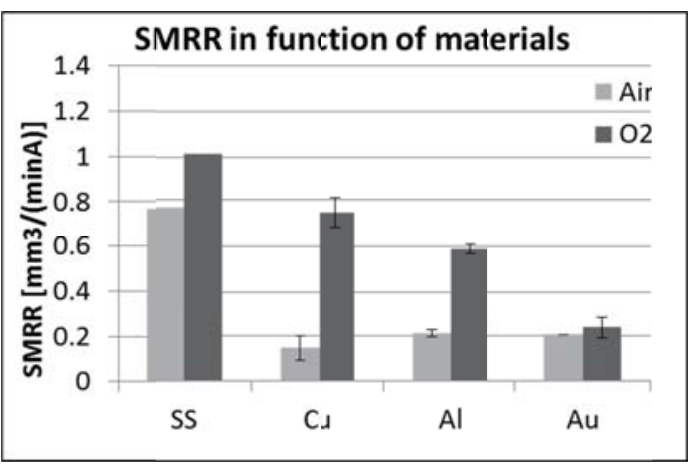

Fig. 10. Current specific material removal rate in function of materials and different gases.

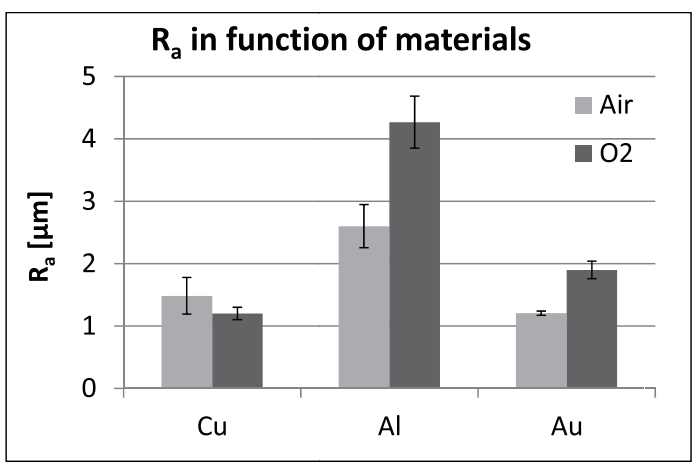

Fig. 11. Surface roughness in function of materials and different gases.

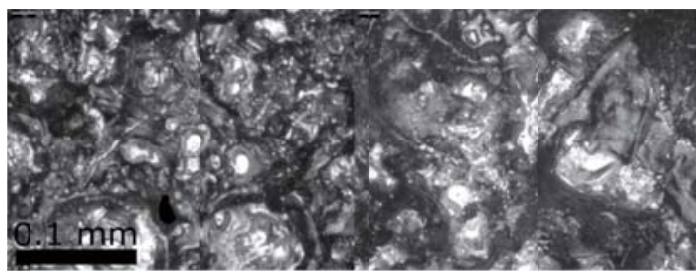

Fig. 12. Surface of dry eroded aluminum with air (left) and oxygen (right) as flushing gas.

\subsubsection{Surface Roughness}

In Fig. 11 the measurements of the surface roughness $R_{a}$ in function of the different work piece materials and flushing gas are showed. The values of gold and copper don't seem to vary much in function of the flushing gas. Copper even seems to have a slightly lower roughness when eroded with oxygen although the difference is not significant. The only material that shows a bigger difference in function of the flushing gas is Aluminum. The difference in surface roughness measured for Aluminum may be caused by a stronger oxidation of the molten work piece material in comparison to the other materials which leads to deeper craters resulting in bigger $R_{a}$.

In Fig. 12 the surface of DEDM machined aluminum with air and oxygen as flushing gases can be observed 
(same scale for both images). It is clearly visible that the erosion with oxygen produces surface with bigger structures than the erosion with air. This goes along with the bigger values of surface roughness due to bigger craters and fits with the fact that the enhancement of the $M R R$ in aluminum caused by oxygen in comparison to air, seems to be caused mainly by a greater removal per spark and not by a greater discharge frequency.

\section{Discussion}

\subsection{On the Influence of the Anode Material}

The results shown in section 4.1 on material removal rate in the DEDM experiments show a clear influence of the anode material. In traditional EDM this behavior is expected and mostly depends on the different physical properties of the eroded material, especially on heat conductivity and melting temperature. The reason of this dependency is that a given energy input of a spark will lead to different craters on the work piece due to the different physical work piece material properties. The specific material removal rate for DEDM of the different material combination shown in section 4.1 is nearly not affected by the different anode materials. In other words the removal per spark is not changing significantly in function of the work piece material. This means that what significantly changes the process are not physical properties as heat conduction coefficient or melting temperature, which show their effect when a discharge occurs, but more likely material properties which influence the stability of the process and therefore the discharge frequency of effective sparks. The comparison with the measurement in oil shows that also in oil EDM the influence of the materials is obviously present, but it is completely different.

In traditional EDM particles in the dielectric fluid can have a great influence on the gap distance and the discharge behavior. So also the different physical properties of the eroded particles of the work piece materials can affect the erosion process leading to frequently short circuiting. As reported also in [10] the SS304 used as work piece is an austenitic and therefore nonmagnetic stainless steel. The observed debris in case of oxygen as flushing gas has ferromagnetic properties and is mainly not conductive. These properties suggest that the main content of the debris eroded with oxygen is iron(II,III) oxide. For cemented carbide it was not possible to collect any significant amount of debris, anyway supposing the debris are oxidized, mostly to $\mathrm{CO}_{2}$ and tungsten oxide may be expected from the erosion. But no tungsten(VI) oxide occurred, as no yellow debris has been observed, tungsten(IV) oxide with the chemical formula $\mathrm{WO}_{2}$ may constitute the main part of the debris. This oxide has the particularity to be a good conductor. The effect that these properties can have on the stability of the process, will be discussed further in section 5.2.

\subsection{Influence of the Anode Material in Combination with the Flushing Gas}

In section 4.2 it is clearly shown that besides the influence of the anode material the flushing gas can have a major influence on the stability of the process and on the removal per spark. The results also show that anode materials exist, that are not affected by different oxygen contents in the flushing gas (i.e. gold). This shows that the combination of the work piece material and the flushing gas is the crucial factor of the DEDM process and as discussed briefly in section 4.2 , it affects the process mainly in two ways. Oxygen enhances the discharge frequency of effective sparks due to a more stable process with less short circuiting. Oxygen also enhances the removal per spark. It seems to be obvious that the discussed effects are related to the chemical reactions between the flushing gas and the work piece material. The two effects will be discussed in more detail in section 5.2.1 and 5.2.2 respectively.

\subsubsection{Removal per Spark}

Regarding the enhancement of the removal per spark in function of the oxygen content in flushing gas it is reasonable, as Kunieda and Furuoya already suggested in [5], that the heat energy released during the oxidation of the eroded material contributes to melt even more material enhancing the removal per spark. On the other hand it is also reasonable that these eroded and oxidized particles have different physical properties and cannot really reattach to the work piece, thus enhancing the flushing efficiency of the sparks. This leads to the same macroscopic effect of more removal per discharge. These two effects can act together with different proportions for different work piece material.

\subsubsection{Stability of the Process}

It may be suggested that different gases lead to different discharge conditions due to different properties as for example the dielectric strength. But as already suggested from Roth et al. in [11] using traditional EDM generators, where the maximum open voltage is around $250 \mathrm{~V}$, the minimum of the Paschen's diagram for a gas discharge cannot be reached. In the Paschen's diagram of the applied gases the minimum breakdown voltage is over $300 \mathrm{~V}$. Nevertheless sparks occur when the working gap gets small enough. In the last years several efforts have been made to characterize electrical breakdown over micro gaps because of their applications in micro electro mechanical systems. It has been shown that in case of gaps less than $5 \mu \mathrm{m}$ in atmospheric air and with at least one metallic electrode there is a discrepancy with the Paschen's curve [12]. A linear dependency between gap distance and breakdown voltage is observed till the Paschen's curve is reached. In fluid or in larger gas filled gaps an electron avalanche triggers the breakdown, ionizing the molecules present in the gap. But in case of very small gas filled gaps the free path of gas 
molecules is too long in comparison to the gap distance. Only few molecules will be present in the gap. The reason for the increasing breakdown voltage of Paschen's curve for very small gaps is the lack of gas molecules. In case of at least one metallic electrode, and gaps, that are small enough $(<5 \mu \mathrm{m})$, an electron field emission can trigger the discharge at lower voltages than the electron avalanche through the gas would do [13]. This mechanism is more similar to a vacuum discharge than a gas discharge. This means that the ignition mechanism of the DEDM discharge is not the same as the one known from traditional EDM in oil or deionized water and it is not directly depending on the flushing gas. Supporting this evidence there are also several spectroscopic measurements where in the case of DEDM only metallic plasma and no lines of the gaseous species have been observed. Kanmani Subbu et al. [14] measured the light emission of sparks during DEDM and evaluated plasma temperature and electrode density using the line pair method. In this publication images of several spectral measurements are shown and it is clearly visible that there is mostly a metal plasma and not gas atoms scattering the light. On the other hand Descoudres [15] published spectral measurements of EDM plasma in deionized water. The results show a spectrum where hydrogen and oxygen are the principal lines. Last but not least in this work erosion of gold work piece with copper electrode flushing with air and oxygen has been presented and no significant difference has been noted between the two gases. This means that there is no influence on the discharge behavior of the process as long as the gas has no chemical reaction with the work piece material. Therefore the influence on the stability of the process can't be ruled by different gas properties such as different dielectric strengths.

Further on, it might be suggested, that the difference of the electron field emission of different oxide film thicknesses play a role in the stability of the process in function of the flushing gas. In fact, as Haylen et al. showed in [16], a thicker oxide film on a copper electrode leads to an enhancement of the number of the field emitted electrons. For the DEDM process this could mean a larger working gap for unchanged electrical conditions and therefore less short circuiting and higher burning voltages. Nevertheless this effect should be visible also in the erosions with gold electrode since it acts on the negative charged copper tool electrode. So also this hypothesis is confuted.

It has been suggested from Roth et al. in [10] and [11] that the properties of the debris particles flushed through the working gap might play a significant role on the stability of the process. The hypothesis consider essentially that if the eroded particles are electrically conductive the gap will be hard to control and the servo regulation won't be able to avoid short circuiting, but if the same particles are insulating they won't disturb the servo regulation and they might even avoid short circuiting preventing the tool electrode from touching directly the work piece. The results presented here support this hypothesis after it was achieved to isolate this effect from the additional heat energy from the chemical reaction. First of all the erosion on gold work piece shows that the effect only appears when the metal can be oxidized. Copper and steel show clearly that when more oxygen is present in the gap short circuiting is decreasing. Also aluminum shows the same pattern but it is not really significant. The small effect observed for aluminum is probably caused by the fact that the process shows a small amount of short circuiting already flushing with air, so no big enhancement can be reached with more oxygen in the flushing gas. The evidence that there is still a significant enhancement of the absolute $M R R$ due to oxygen suggests that there is significant chemical reaction going on releasing heat energy and/or still changing the properties of the particles. The difference of the aluminum in respect to the other measured elements in this work may still be due to the electrical conductivity of the debris particle. In fact the most metallic nitrides are conductive but the aluminum nitride is a semiconductor. For this reason it may insulate the working gap in a similar manner as the aluminum oxide does. In the same way also the difference on the stability of the steel and the cemented carbide erosions of section 4.1 may be explained with the difference of the debris particles' properties discussed in section 5.1. These possible explanations of the influence on the $M R R$ of work piece material and flushing gas also fit with the fact that the tool electrode material has only a poor influence on the MRR. Since only a few amount of tool material is removed and will be in the erosion gap no influence is expected.

\section{Conclusion}

A comparison of material removal rate and tool wear ratio of dry electrical discharge machining with different tool electrode, work piece materials and flushing gases at two fixed EDM parameters has been presented. The results have been compared to the discharge behavior. The comparison shows that influence on the material removal rate depends strongly on the combination of the flushing gas and the work piece material. To consider either flushing gas or work piece material doesn't suffice to predict the behavior of the erosion. The results presented permit to deduce two main effects on the material removal rate. The first one enhances the removal per spark while the second one changes the process stability meaning and thus the effective discharge frequency. The different work piece materials also show different contributions of the two effects in the overall effect on the absolute material removal rate. The enhancement of the removal per spark is reasonably explained by the additional energy released from chemical reaction with the gas or the different properties of the debris that lead to more or less reattachment. The enhancement of the effective discharge frequency seems to be related to the electrical properties of the debris particles. The two hypotheses that gases may influence directly the discharge behavior or that the different electron field emission due to different copper ox- 
ide films on the electrode may change the stability of the process have been confuted by the evidence of the erosion with gold work piece and their comparison with other materials. For this reason to gain better insight into the physics of the EDM process and eventually an enhancement of the DEDM process' performance the chemical interaction between work piece and flushing gas and the resulting properties of the debris particles have to be considered. Further experiments to better understand the ruling mechanisms of DEDM are running and especially efforts to support the presented hypothesis by quantitative prediction are ongoing.

\section{Acknowledgements}

We would like to thank Marco Boccadoro from AGIE-Charmilles, Mario Graf from Carbagas and Dr. Christoph Hollenstein from the center of plasma physics research of the Federal Technical University of Lausanne for the great collaboration and CTI for funding the research.

\begin{tabular}{lll}
\multicolumn{2}{l}{ Nomenclature } & \\
$M R R$ & Material removal rate & {$\left[\mathrm{mm}^{3} / \mathrm{min}\right]$} \\
$S M R R$ & Current specific material removal rate & {$\left[\mathrm{mm}^{3} /(\mathrm{minA})\right]$} \\
$T W R$ & Tool wear ratio & {$[\%]$} \\
$U$ & Open voltage & {$[\mathrm{V}]$} \\
$U_{B}$ & Burning voltage & {$[\mathrm{V}]$} \\
$I$ & Current & {$[\mathrm{A}]$} \\
$I_{a}$ & Time averaged current & {$[\mathrm{A}]$} \\
$T_{S}$ & Spark time & {$[\mu \mathrm{s}]$} \\
$T_{P}$ & Pause time & {$[\mu \mathrm{s}]$} \\
$p$ & Flushing pressure & {$[\mathrm{bar}]$} \\
$\tau_{A}$ & Relative arcing time & {$[\%]$} \\
$\tau_{S}$ & Relative sparking time & {$[\%]$} \\
$\mathrm{CC}$ & Cemented Carbide & - \\
$\mathrm{SS}$ & Stainless Steel & - \\
$\Delta l$ & Length wear of the tool & {$[\mathrm{mm}]$} \\
$A_{T}$ & Cross section area of the tool & {$\left[\mathrm{mm}{ }^{2}\right]$} \\
$R_{a}$ & Surface roughness & {$[\mu \mathrm{m}]$}
\end{tabular}

\section{References:}

[1] B. Schumacher, "After 60 years of EDM the discharge process remains still disputed," J. of Materials Processing Technology, Vol.149, pp. 376-381, 2004.

[2] M. Kunieda, Y. Miyoshi, T. Takaya, N. Nakajima, Y. ZhanBo, and M. Yoshida, "High speed 3D milling by dry EDM," Cirp annals, manufacturing technology, Vol.52, Issue 1, pp. 147-150, 2003.

[3] Z. B. Yu, T. Jun, and M. Kunieda, "Electrical discharge machining of cemented carbide," J. of Materials Technology, Vol.149, pp. 353$357,2004$.

[4] M. Kunieda and M. Yoshido, "Electrical discharge machining in gas," Cirp annals, manufacturing technology, Vol.46, Issue 1, pp. 143-146, 1997.

[5] M. Kunieda, S. Furuoya, and N. Taniguchi, "Improvement of EDM efficiency by supplying oxygen gas into gap," CIRP Annals - Manufacturing Technology, Vol.40, pp. 215-218, 1991.

[6] M. Kunieda, T. Talaya, and S. Nakano, "Improvement of Dry EDM characteristics using piezoelectric actuator," CIRP Annals - Manufacturing Technology, Vol.53, pp. 183-186, 2004.

[7] Q. H. Zhang, R. Du, J. H. Zhang, and Q. B. Zhang, "An investigation of ultrasonic-assisted electrical discharge machining in gas," Int. J. of Machine Tools \& Manufacture, Vol.46, pp. 1582-1588, 2006.
[8] S. Joshi, P. Govindan, A. Malshe, and K. Rajurkar, "Experimental characterization of dry EDM performed in a pulsating magnetic field," CIRP Annals - Manufacturing Technology, Vol.60, Issue 1, pp. 239-242, 2011.

[9] N. Abbas, D. Solomon, and Md. Bahari, "A review on current research trends in electrical discharge machining (EDM)," Int. J. of Machine Tools \& Manufacture, Vol.47, pp. 1214-1228, 2007.

[10] R. Roth, F. Kuster, and K. Wegener, "Influence of Oxidizing Gas on the Stability of Dry Electrical Discharge Machining Process," Procedia CIRP, Vol.6, pp. 339-344, 2013.

[11] R. Roth, H. Balzer, F. Kuster, and K. Wegener, "Influence of the Anode Material on the Breakdown Behavior in Dry Electrical Discharge Machining," Procidia CIRP, Vol.1, pp. 639-644, 2012.

[12] T. Ono, D. Y. Sim, and M. Esashi, "Micro discharge and elctric breakdown in a micro-gap," J. micromech. microeng., Vol.10, pp. 445-451, 2000.

[13] M. Klas, S. Matejcik, and B. Radjenovic, "Experimental and theoretical studies of the breakdown voltage characteristic at micrometre separetion in air," a Letters J. Exploring the Frontiers of Physics, EPL 95, 2011.

[14] S. Kanmani Subbu, G. Karthikeyan, J. Ramkumar, and S. Dhamodaran, "Plasma characterisation of dry $\mu$-EDM," Int. J. Adv. Manuf. Technol. DOI 10.1007/s00170-011-3162-4, 2011.

[15] A. Descoudres, "Characterisation of electrical discharge machining plasmas," Thèse $\mathrm{N}^{\circ} 3542$, Ecole polytechnique fédérale de Lausanne, 2006.

[16] A. Heylen, A. Guile, and D. Morgan, "Electron field emission from copper with various thicknesses of oxide film," IEE Proc., Vol.131, Pt. A. No.2, Mar. 1984.

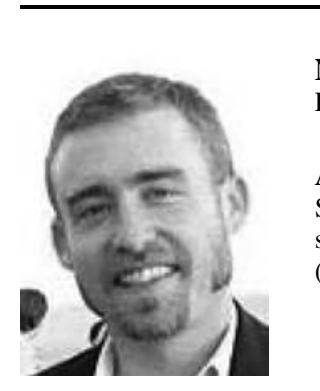

Name:

Raoul Roth

\section{Affiliation:}

Scientific Collaborator and Ph.D. Student at Institute for Machine Tools and Manufacturing (IWF) of ETH Zürich

Address:

CLA G4, Tannenstrasse 3, 8092 Zürich, Switzerland

Brief Biographical History:

2007- Degree in Mechanical Engineering at ETH Zürich

2007-2009 Scientific Collaborator at Inspire AG of ETH Zürich

2009-2013 Scientific Collaborator at IWF of ETH Zürich

Main Works:

- R. Roth, F. Kuster, and K. Wegener, "Influence of Oxidizing Gas on the Stability of Dry Electrical Discharge Machining Process," Seventeenth CIRP Conf. on Electro Physical and Chemical Machining, 2013, Leuven, Belgium, Vol.6, pp. 338-343.

- R. Roth, H. Balzer, F. Kuster, and K. Wegener, "Influence of the Anode Material on the Breakdown Behavior in Dry Electrical Discharge Machining," 5th CIRP Conf. on High Performance Cutting 2012, Zürich, Switzerland, Vol.1, pp. 639-644, 2012. 


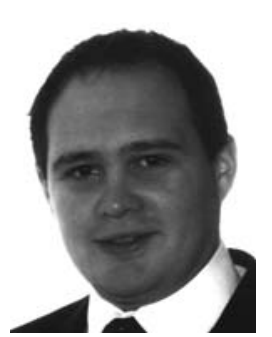

Name:

Beck Lukas

\section{Affiliation:}

Student, ETH Zürich

Address:

Bachweidstrasse 20, 9011 St. Gallen, Switzerland

Brief Biographical History:

2011- Student at ETH Zürich in Mechanical Engineering

Main Works:

- B. Lukas, "Trockene Funkenerosion - Einfluss der Oxidation," Term Paper at IWF of ETH Zürich.

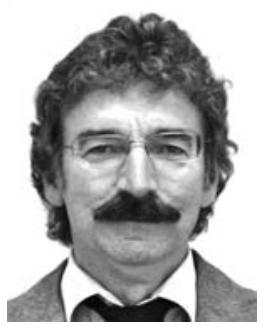

\section{Name:}

Hartmi Balzer

\section{Affiliation:}

BALZER-TECHNIK SA

Address:

Rte de l'Industrie 65, 1564 Domdidier, Switzerland

Brief Biographical History:

1986- founding of the company BALZER-TECHNIK SA and invention of the electrodes in tungsten carbide

1997- invention of the trefoil and multichannel tubular electrodes in tungsten carbide

\section{Main Works}

- Specialist in micro erosion and accessories

- test pins in tungsten carbide

- female test pins in tungsten carbide

- electrodes in tungsten carbide from $\varnothing 0.050$ and $480 \mathrm{~mm}$ length

Membership in Academic Societies:

- the Castel Carignan Holding, Administrator and owner

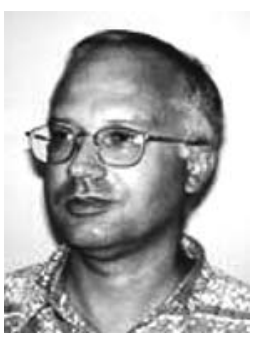

\section{Name: \\ Friedrich Kuster}

\section{Affiliation:}

Dr., Sc., Techn., Head of the Research Team for Manufacturing Processes at the Institute of Machine Tools and Manufacturing of the ETH Zurich

Address:

CLA G17.1, Tannenstrasse 3, 8092 Zürich, Switzerland

Brief Biographical History:

1980-1987 Scientific Research Assistant at IWF in vibration group 1987-1997 Ph.D. Student at IWF of ETH Zürich

1987-Head of Research Team for Vibrations at IWF

1997-2000 Head of Research Team for Vibrations and Machine Tools at IWF

2000-2003 Scientific Coordinator at IWF

2003- Head of the Research Team for Manufacturing Processes at the Institute of Machine Tools

\section{Main Works:}

- F. Kuster, "Bohrstangendynamik: faserverstärkte Materialien und Beschichtungen im Vergleich zu Hilfsmassensystemen," Doctoral Thesis, 1993.

Membership in Academic Societies:

- the International Academy for Production Engineering (CIRP),

Corporate Member

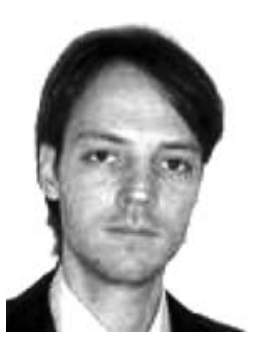

\section{Name:}

Eduardo Weingärtner

Affiliation:

Dr., Scientific Collaborator at Inspire AG of ETH Zürich

Address:

CLA G4, Tannenstrasse 3, 8092 Zürich, Switzerland

\section{Brief Biographical History:}

2005- Master Mechanical Engineering at Federal University of Santa Catarina (UFSC), Brasilien

2007-2013 Scientific Collaborator and Ph.D. Student at Institute for Machine Tools and Manufacturing (IWF) of ETH Zürich

2013- Scientific Collaborator at Inspire AG of ETH Zürich

\section{Main Works:}

- E. Weingärtner, S. Jaumann, F. Kuster, and K. Wegener, "On-machine wire electrical discharge dressing (WEDD) of metal-bonded grinding wheels," The Int. J. of Advanced Manufacturing Technology, 2010, ISSN 0268-3768, Band 49, Nr. 9-12, Seiten 1001-1007.

- E. Weingärtner, "On-machine wire electrical discharge dressing of metal bonded grinding wheels," Doctor Thesis at IWF of ETH Zürich, 2013.

Membership in Academic Societies:

- the International Academy for Production Engineering (CIRP), Research Affiliate 


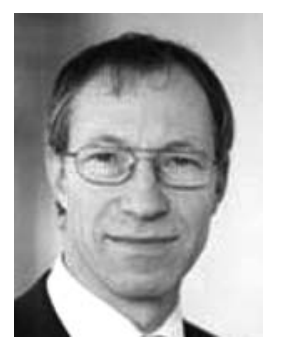

Name:

Konrad Wegener

\section{Affiliation:}

Prof., Dr., Head of Institute for Machine Tools and Manufacturing ETH Zürich

Address:

CLA G4, Tannenstrasse 3, 8092 Zürich, Switzerland

Brief Biographical History:

1991- Ph.D. in Mechanical Engineering TU Braunschweig

1991-1997 Technical University Braunschweig: Lectures in "Tensor

Calculus for Engineers I and II," Leading Engineers to Concepts of

Nonlinear Continuum Mechanics

1997- Call from TH Darmstadt as Professor for Production and Forming

Technology (Succession Prof. Schmoeckel)

1997-1999 TH Darmstadt: Lectures in "Forming Machines I and II"

"Forming technology I and II"

1999-2003 CEO Schuler Held Lasertechnik, Heusenstamm Company of 50 Employees, Dealing with Special Purpose Machinery for Cutting and Welding for the Automotive, the Shipbuilding and the Aircraft Industry 2003-2011 CEO of Inspire AG for Mechatronic Production Systems and Manufacturing, a Technology Transfercenter at ETH Zürich Leading a Group of 62 Persons, 26 Ph.D. Students and 3 Ph.D., Annual Research Budget (Turnover): 7.6 Mio CHF (2010)

2003- Head of Institute for Machine Tools and Manufacturing ETH Zürich Leading a Group of 25 Persons, 16 Ph.D. Students, 3 Ph.D. Research in

Machine Tools, Production Processes, Annual Research Budget 1.7 Mio

CHF

Main Works:

- http://www.iwf.mavt.ethz.ch/people/staff/index/

Wegen_Konra_122701319200486/index

Membership in Academic Societies:

- the International Academy for Production Engineering (CIRP),

Associate and Corporate Member

- International Journal of Automation Technology (IJAT), Editorial Board

- International Journal of Mechatronics and Manufacturing Systems

(IJMMS), Editorial Board 